\title{
SOME COMPOUNDS FROM FLOWER OF WEDELIA TRILOBATA (L.) HITCH.
}

\section{(ASTERACEAE)}

\author{
Truong Thi Hong Nguyet, Nguyen Thi Hoai Thu, \\ Ton That Quang, Nguyen Kim Phi Phung \\ University of Science, VNU HCM \\ (Manuscript Received on January $24^{\text {th }} 2011$, Manuscript Revised October $25^{\text {th }}$, 2011)
}

ABSTRACT: Wedelia trilobata Hitch. has not much been chemically and biologically studied. From the flower of Wedelia trilobata, ten compounds were isolated: friedelan-3 $\beta$-ol (or epifriedelanol)

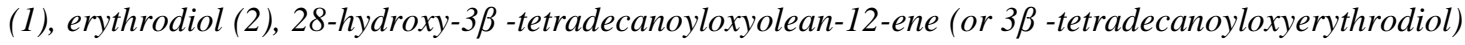

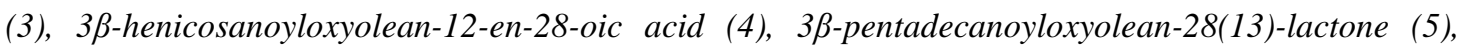
kaura-9(11),16-dien-18-oic acid (or grandiflorenic acid) (6), 2-(3-hydroxy-3,7,11,15tetramethylhexadecyl)-3,5,6-trimethyl-1,4-dihydroxybenzene (or tocopherolhydroquinone) gramisterol (8), stigmasterol (9) and $\beta$-sitosterol 3-O- $\beta$-D-glucopyranoside (10). The structures of these compounds were determined through the interpretation of their NMR data and compared with references.

Key words: Asteraceae, Wedelia trilobata, triterpenoids, diterpenoids, sterols.
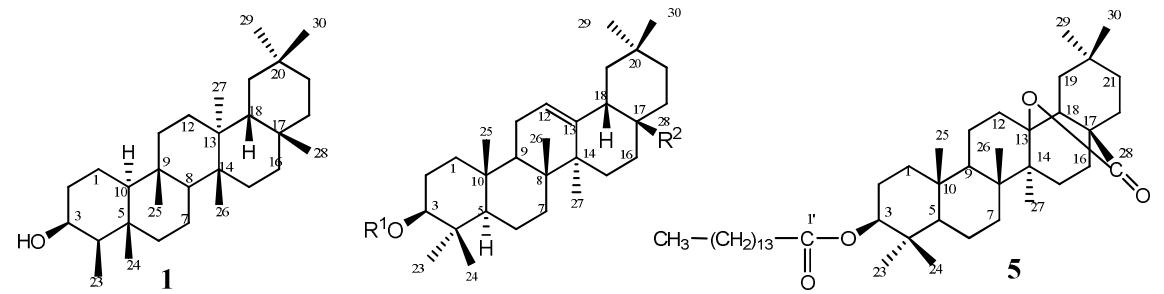

2. $\mathrm{R}^{1}=\mathrm{H} ; \mathrm{R}^{2}=\mathrm{CH}_{2} \mathrm{OH}$

3. $\mathrm{R}^{1}=\mathrm{CO}\left(\mathrm{CH}_{2}\right)_{12} \mathrm{CH}_{3} ; \mathrm{R}^{2}=\mathrm{CH}_{2} \mathrm{OH}$

4. $\mathrm{R}^{1}=\mathrm{CO}\left(\mathrm{CH}_{2}\right)_{19} \mathrm{CH}_{3} ; \mathrm{R}^{2}=\mathrm{COOH}$

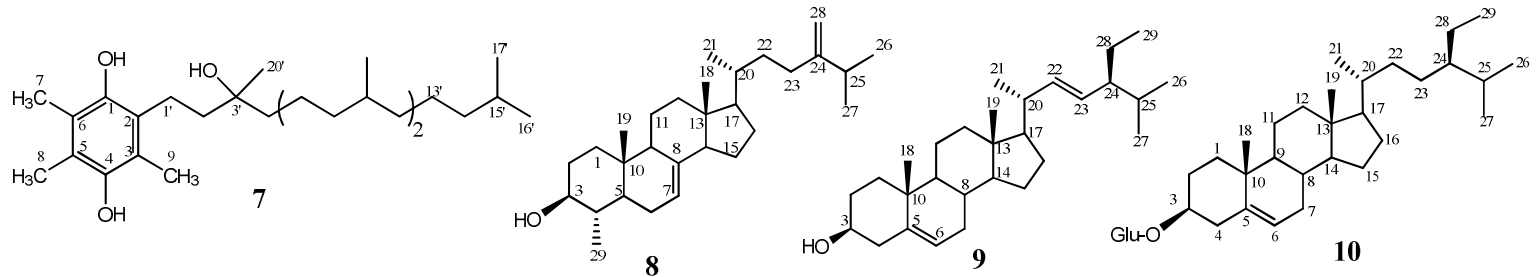

\section{INTRODUCTION}

Wedelia trilobata Hitch. is a plant of Asteraceae family that wildly grows in many areas in Vietnam. This herb was used as folk remedy for haemostatic, treating diarrhea, snake bite, wound, ect. ${ }^{[1]}$ Moreover, its flowers have not much been chemically and biologically studied, therefore they were chosen to study. In this paper, we report the 
isolation and structural determination of ten compounds: friedelan-3 $\beta$-ol (or epifriedelanol) (1), erythrodiol (2), 28-hydroxy-3 $\beta$ tetradecanoyloxyolean-12-ene (or $3 \beta$ tetradecanoyloxyerythrodiol) (3), 3ßhenicosanoyloxyolean-12-en-28-oic acid (4), $3 \beta$-pentadecanoyloxyolean-28(13)-lactone (5), kaura-9(11),16-dien-18-oic acid (or grandiflorenic acid) (6), 2-(3-hydroxy3,7,11,15-tetramethylhexadecyl)-3,5,6trimethyl-1,4-dihydroxybenzene (or tocopherolhydroquinone) (7), gramisterol (8), stigmasterol (9) and $\beta$-sitosterol 3-O- $\beta$-Dglucopyranoside (10).

\section{EXPERIMENTAL}

\section{General}

The NMR spectra were measured on a Bruker Avance spectrometer, at $500 \mathrm{MHz}$ for ${ }^{1} \mathrm{H}$ and $125 \mathrm{MHz}$ for ${ }^{13} \mathrm{C}$, in the Institute of Chemistry, Vietnam Academy of Science and Technology. The HR-ESI-MS were recorded on a HR-ESI-MS MicroOTOF-Q mass spectrometer in the University of Science, National University - HCM City.

\section{Plant material}

Flowers of plant were collected in Ho Chi Minh city, Vietnam in November 2008. The scientific name of plant was identified by pharmacist Phan Duc Binh, Associate Editorin-Chief of the Journal of Drug and Health. A voucher specimen (No US-B002) was deposited in the herbarium of the Department of Organic Chemistry, University of Science, National University - Ho Chi Minh City.

\section{Extraction and isolation}

Fresh flower $(10.3 \mathrm{~kg})$ was washed, dried, ground into powder (950g) and extracted by maceration with ethanol at room temperature then the extracted solution was evaporated in reduced pressure to give methanol residue (302.6g). This crude extract was suspended in water and partitioned against petroleum ether to afford petroleum ether residue (E, $70 \mathrm{~g}$ ) and water residue. The $\mathbf{E}$ residue was subjected to silica gel column chromatography, eluted with gradient solvent system of petroleum ether: chloroform (10: 0 to $0: 10$ ) to obtain $\mathbf{1 , 3 , 4 , 5}$, 7, 8 and 9, then chloroform:methanol (10: 0 to 0:10) to afford 2, 6 and $\mathbf{1 0 .}$

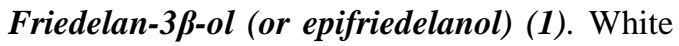
crystal, mp. $268-270^{\circ} \mathrm{C}\left(\mathrm{CHCl}_{3}\right)$. The ${ }^{1} \mathrm{H}-\mathrm{NMR}$, $\mathrm{CDCl}_{3}, \delta$ ppm: 3.73 (1H, s, H-3), 0.93 (3H, s, H-23), 0.96 (3H, s, H-24), 0.86 (3H, s, H-25), 0.99 (3H, s, H-26), 1.00 (3H, s, H-27), 1.17 (3H, s, H-28), 0.99 (3H, s, H-29), 0.94 (3H, s, $\mathrm{H}-30$ ). The ${ }^{13} \mathrm{C}-\mathrm{NMR}, \mathrm{CDCl}_{3}$, pppm (Table 1).

Erythrodiol (2). White powder, mp. $164-166^{\circ} \mathrm{C}$ $\left(\mathrm{CHCl}_{3}\right)$. The ${ }^{1} \mathrm{H}-\mathrm{NMR}, \mathrm{CDCl}_{3}, \delta$ ppm: 5.19 (1H, $t, 4.0, \mathrm{H}-12), 3.22$ (1H, $d, 11.0, \mathrm{H}-3), 3.22$ (1H, $d, 11.0, \mathrm{H}-28 \mathrm{a}), 3.55$ (1H, $d, 11.0, \mathrm{H}-28 \mathrm{~b})$, 0.99 (3H, s, H-23), 0.79 (3H, s, H-24), 0.93 (3H, s, H-25), 0.94 (3H, s, H-26), 1.16 (3H, s, H-27), 0.88 (3H, s, H-29), 0.87 (3H, s, H-30). The ${ }^{13} \mathrm{C}-\mathrm{NMR}, \mathrm{CDCl}_{3}$, $\delta$ ppm (Table 1).

\section{8-Hydroxy-3 $\beta$-tetradecanoyloxyolean-12-ene}

(3). White powder, mp. $127-128^{\circ} \mathrm{C}\left(\mathrm{CHCl}_{3}\right)$. The ${ }^{1} \mathrm{H}-\mathrm{NMR}, \mathrm{CDCl}_{3}$, $\delta$ ppm: $5.19(1 \mathrm{H}, t, 3.5$, $\mathrm{H}-12), 4.50(1 \mathrm{H}, m, \mathrm{H}-3), 3.21(1 \mathrm{H}, d, 11.0, \mathrm{H}-$ 
28a), 3.54 (1H, $d, 11.0, \mathrm{H}-28 \mathrm{~b}), 0.86$ (3H, $s, \mathrm{H}-$ 23), 0.86 (3H, s, H-24), 0.96 (3H, $s, \mathrm{H}-25)$, $0.94(3 \mathrm{H}, s, \mathrm{H}-26), 1.16(3 \mathrm{H}, s, \mathrm{H}-27), 0.89$ (3H, s, H-29), 0.87 (3H, s, H-30), 2.29 (2H, $t$, 7.0, H-2'), 0.88 (3H, H-14'). The ${ }^{13} \mathrm{C}-\mathrm{NMR}$, $\mathrm{CDCl}_{3}$, Sppm (table 1).

3及-Henicosanoyloxyolean-12-en-28-oic acid (4). Colorless wax. The ${ }^{1} \mathrm{H}-\mathrm{NMR}, \mathrm{CDCl}_{3}$, Sppm: $5.27(1 \mathrm{H}, t, 3.5, \mathrm{H}-12), 4.49(1 \mathrm{H}, d d$, 11.0, 6.0, H-3), 0.86 (3H, $s, \mathrm{H}-23), 0.85$ (3H, $s$, H-24), 0.94 (3H, s, H-25), 0.75 (3H, $s, \mathrm{H}-26)$, $1.13(3 \mathrm{H}, s, \mathrm{H}-27), 0.90$ (3H, $s, \mathrm{H}-29), 0.92$ (3H, $s, \mathrm{H}-30), 2.29$ (2H, t, 7.0, H-2'), $0.88(3 \mathrm{H}$, $\left.t, 7.0, \mathrm{H}-21^{\prime}\right)$. The ${ }^{13} \mathrm{C}-\mathrm{NMR}, \mathrm{CDCl}_{3}, \delta \mathrm{ppm}$ (Table 1).

3ק-Pentadecanoyloxyolean-28(13)-lactone (5). White powder, mp. $241-243^{\circ} \mathrm{C}\left(\mathrm{CHCl}_{3}\right)$. The ${ }^{1} \mathrm{H}-\mathrm{NMR}, \mathrm{CDCl}_{3}$, $\delta \mathrm{ppm}: 4.48(1 \mathrm{H}, d d, 10.0$, 6.5, H-3), 0.85 (3H, $s, \mathrm{H}-23), 0.85$ (3H, $s, \mathrm{H}-$ 24), 0.89 (3H, $s, \mathrm{H}-25), 1.15(3 \mathrm{H}, s, \mathrm{H}-26)$, $1.04(3 \mathrm{H}, s, \mathrm{H}-27), 0.99$ (3H, s, H-29), 0.86 (3H, $s, \mathrm{H}-30), 2.29$ (2H, $t, 7.0, \mathrm{H}-2$ '), 0.88 (3H, H-15'). The ${ }^{13} \mathrm{C}-\mathrm{NMR}, \mathrm{CDCl}_{3}$, $\delta$ ppm (Table 1).

Kaura-9(11),16-dien-18-oic acid (or grandiflorenic acid) (6). White crystal, mp. $155-157^{\circ} \mathrm{C}\left(\mathrm{CHCl}_{3}\right)$. The ${ }^{1} \mathrm{H}-\mathrm{NMR}, \mathrm{CDCl}_{3}$, Sppm: $5.23(1 \mathrm{H}, d d, 3.5,3.0, \mathrm{H}-11), 4.91(1 \mathrm{H}$, d, 1.0, H-17a), 4.79 (1H, s, H-17b), 2.77 (1H, brs, H-13), 1.24 (3H, s, H-19), 1.02 (3H, s, H20). The ${ }^{13} \mathrm{C}-\mathrm{NMR}, \mathrm{CDCl}_{3}, \delta$ ppm (Table 1).

2-(3-Hydroxy-3,7,11,15-

tetramethylhexadecyl)-3,5,6-trimethyl-1,4dihydroxybenzene (7). Colorless oil. The ${ }^{1} \mathrm{H}$ NMR, $\mathrm{CDCl}_{3}, \delta$ ppm: $2.10(3 \mathrm{H}, s, \mathrm{H}-7), 2.15$
(3H, s, H-8), 2.10 (3H, s, H-9), 1.22 (3H, s, H$\left.20^{\prime}\right), 0.87$ (6H, $d, 7.0, \mathrm{H}-16$ ', H-17'), 0.56 (3H, $\left.d, 4.5, \mathrm{H}-18^{\prime}\right), 0.43$ (3H, $\left.d, 4.5, \mathrm{H}-19 '\right)$. The

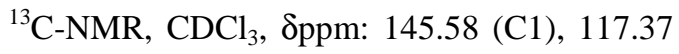
(C2), 118.48 (C3), 144.55 (C4), 121.03 (C5), 122.63 (C6), 11.77 (C7), 12.20 (C8), 11.27 (C9), 20.77 (C1'), 31.58 (C2'), 74.53 (C3’), 39.84 (C4'), 21.05 (C5'), 37.44 (C6'), 32.72 (C7'), 37.49 (C8'), 24.46 (C9'), 37.30 (C10'), 32.81 (C11'), 37.47 (C12'), 24.81 (C13'), 39.39 (C14'), 27.99 (C15'), 22.63 (C16'), 22.72 (C17'), 19.67 (C18'), 19.76 (C19'), 23.81 (C20’).

Gramisterol (8). White crystal, mp. $162-163^{\circ} \mathrm{C}$ $\left(\mathrm{CHCl}_{3}\right)$. The ${ }^{1} \mathrm{H}$ and ${ }^{13} \mathrm{C}-\mathrm{NMR}$ data of $\mathbf{8}$ were good compatibility with the ones in literature ${ }^{[5]}$. The ${ }^{1} \mathrm{H}-\mathrm{NMR}, \mathrm{CDCl}_{3}$, $\delta$ ppm: $5.17(1 \mathrm{H}, m, \mathrm{H}-$ 7), $4.65(1 \mathrm{H}, d, 1.0, \mathrm{H}-28 \mathrm{a}), 4.71(1 \mathrm{H}, s, \mathrm{H}-$ 28b), 0.53 (3H, $s, \mathrm{H}-18), 0.82$ (3H, $s, \mathrm{H}-19)$, $0.95(3 \mathrm{H}, d, 6.5, \mathrm{H}-21), 1.03$ (3H, $d, 7.0, \mathrm{H}-26)$, $1.02(3 \mathrm{H}, d, 6.5, \mathrm{H}-27), 0.98$ (3H, $d, 6.0, \mathrm{H}-29)$. The ${ }^{13} \mathrm{C}-\mathrm{NMR}, \mathrm{CDCl}_{3}$, $\delta$ ppm (Table 1).

Stigmasterol (9). White needle crystal, mp. $164-166^{\circ} \mathrm{C}\left(\mathrm{CHCl}_{3}\right)$. The ${ }^{1} \mathrm{H}$ and ${ }^{13} \mathrm{C}-\mathrm{NMR}$ data of 9 were good compatibility with the ones in literature ${ }^{[5]}$. The ${ }^{1} \mathrm{H}-\mathrm{NMR}, \mathrm{CDCl}_{3}, \delta \mathrm{ppm}$ : $5.34(1 \mathrm{H}$, brs, H-6), $5.16(1 \mathrm{H}, d d, 15.0,8.5, \mathrm{H}-$ 22), $5.02(1 \mathrm{H}, d d, 15.0,9.0, \mathrm{H}-23), 3.51(1 \mathrm{H}$, $t t, 11.0,5.0, \mathrm{H}-3), 1.01$ (3H, s, H-18), 0.70 (3H, $s, \mathrm{H}-19), 1.03$ (3H, $d, 6.0, \mathrm{H}-21), 0.79(3 \mathrm{H}, d$, 7.0, H-26), 0.84 (3H, $d, 6.5, \mathrm{H}-27), 0.81(3 \mathrm{H}, t$, 6.0, H-29). The ${ }^{13} \mathrm{C}-\mathrm{NMR}, \mathrm{CDCl}_{3}$, $\delta$ ppm (Table 1).

B-Sitosterol 3-O- $\beta$-D-glucopyranoside (10). White powder, mp. $284-286^{\circ} \mathrm{C}(\mathrm{MeOH})$. The 
${ }^{1} \mathrm{H}$ and ${ }^{13} \mathrm{C}-\mathrm{NMR}$ data of $\mathbf{1 0}$ were good compatibility with the ones in literature ${ }^{[4]}$. The ${ }^{1} \mathrm{H}-\mathrm{NMR}$, DMSO- $d_{6}$, $\delta$ ppm: $5.31(1 \mathrm{H}, b r d, \mathrm{H}-$ 6), $3.62(1 \mathrm{H}, m, \mathrm{H}-3), 4.83\left(1 \mathrm{H}, d, 5.0, \mathrm{H}-1^{\prime}\right)$. The ${ }^{13}$ C-NMR, DMSO- $d_{6}$, $\delta$ ppm (Table 1).

\section{RESULTS AND DISCUSSION}

Compound (1) was a triterpen with 30 carbons in the ${ }^{13} \mathrm{C}$-NMR spectrum. $\mathbf{1}$ had an oxygenated carbon signal at $\delta 72.7$ of $\mathrm{C}-3$ as normal, which corresponded to an oxygenated methine proton signal at $3.73 \mathrm{ppm}$. Besides that it had 8 singlet methyl proton signals upfield at: $0.86,0.93,0.94,0.96,0.99,0.99,1.00$ and 1.17. So 1 should be a friedelan triterpen by comparison with the published data. ${ }^{[7]}$

Compound (2) was also a triterpen with 30 carbons in the ${ }^{13} \mathrm{C}$-NMR spectrum. 2 had an oxygenated methine carbon signal at $\delta 79.0$ of C-3 as normal, one oxygenated methylene carbon signal at $69.7 \mathrm{ppm}, 7$ singlet methyl proton signals in high field zone of $\delta 0.79$, $0.87,0.88,0.93,0.94,0.99$ and 1.16 and two olefinic carbon signals at $\delta 122.4$ and 142.2 of C-12 and C-13, respectively, in olean-12-ene skeleton. So $\mathbf{2}$ was determined as erythrodiol by comparison with the published data. ${ }^{[7]}$

Compound (3) was a white powder. The NMR spectra data of $\mathbf{3}$ had some signals like $\mathbf{2}$ such as a trisubstituted double bond $\left(\delta_{\mathrm{H}} 5.19 \mathrm{~m}\right.$, $\delta_{\mathrm{C}} 144.2 s, 122.3 d$ ), an oxygenated methine group $\left(\delta_{\mathrm{H}} 4.50, \delta_{\mathrm{C}} 80.6\right)$, an oxygenated methylene group $\left(\delta_{\mathrm{H}} 3.54\right.$ and $\left.3.21, \delta_{\mathrm{C}} 69.7\right)$ and 7 singlet methyl proton signals upfield at $\delta$
0.86, 0.87, 0.87, 0.88, 0.94, 0.96 and 1.16 . However, the hydroxyl group at C-3 was esterified to be -O-CO-R because the HMBC spectrum showed the correlation of proton $\mathrm{H}-3$ $(\delta 4.50)$ with the resonant peak at $\delta 173.1$ $\left(\mathrm{C}=\mathrm{O}, \mathrm{C}-1^{\prime}\right)$. This spectrum also showed the correlation of proton H-2' with C-1'. Proton H2' $(2.29,2 \mathrm{H}, t, 7.0 \mathrm{~Hz})$ confirmed the presence of an acyl group -O-CO-R at C-3. The long side chain ester at C-3 was determined by MS. The ESI-MS (positive mode) showed a molecular ion peak at $\mathrm{m} / z=688.5239$ $\left[\mathrm{M}+\mathrm{H}_{2} \mathrm{O}+\mathrm{NH}_{4}\right]^{+}$corresponding to the molecular formula of $\mathrm{C}_{44} \mathrm{H}_{76} \mathrm{O}_{3}+\mathrm{H}_{2} \mathrm{O}+\mathrm{NH}_{4}$ (Calcd. for $\mathrm{C}_{44} \mathrm{H}_{76} \mathrm{O}_{3}+\mathrm{H}_{2} \mathrm{O}+\mathrm{NH}_{4} \quad$ 688.6244). The aglycone moiety with two hydroxyl groups had the mass of $441 \mathrm{amu}\left(\mathrm{C}_{30} \mathrm{H}_{49} \mathrm{O}_{2}\right)$ so the side chain moiety had the mass of 211 amu. This mass well suited to the alcanoyl group of -CO$\left(\mathrm{CH}_{2}\right)_{13}-\mathrm{CH}_{3}$. So the compound was determined as 28-hydroxy-3 $\beta$-tetradecanoyloxyolean-12ene or $3 \beta$-tetradecanoyloxyerythrodiol via the comparison with the literature. ${ }^{[2]}$

Compound (4) was a colorless wax. The NMR spectra data of $\mathbf{4}$ had some signals like $\mathbf{3}$ such as a trisubstituted double bond $\left(\delta_{\mathrm{H}} 5.27 \mathrm{~m}\right.$, $\delta_{\mathrm{C}} 143.6 s, 122.6 d$ ), an oxygenated methine group $\left(\delta_{\mathrm{H}} 4.49, \delta_{\mathrm{C}} 80.6\right), 8$ singlet methyl proton signals in high field zone and an acyl group -O-CO-R at $\mathrm{C}-3$ via the correlation between $\mathrm{H}-3$ and the resonant peak at $\delta 173.7$ ( $\left.\mathrm{C}=\mathrm{O}, \mathrm{C}-1^{\prime}\right)$. However, 4 had one more carboxyl carbon signal at $\delta 184.0$ of $\mathrm{C}-28$ by the correlations of $\mathrm{H}-18, \mathrm{H}-16, \mathrm{H}-22$ with $\mathrm{C}$ 28. The long side chain ester at C-3 was 
determined by MS. The ESI-MS (positive mode) showed a molecular ion peak at $\mathrm{m} / \mathrm{z}=$ $803.5495[\mathrm{M}+\mathrm{K}]^{+}$corresponding to the formula of $\mathrm{C}_{51} \mathrm{H}_{88} \mathrm{O}_{4}+\mathrm{K}$ (Calcd. for $\mathrm{C}_{51} \mathrm{H}_{88} \mathrm{O}_{4}+\mathrm{K}$ 803.6320), from that, the alcanoyl group shoud be $-\mathrm{CO}-\left(\mathrm{CH}_{2}\right)_{19}-\mathrm{CH}_{3}$. So the compound was determined as 3 $\beta$-henicosanoyloxyolean-12-en28-oic acid via the comparison with the literature. $^{[2]}$

Compound (5) was a white powder. The NMR spectra data of $\mathbf{5}$ had some signals like $\mathbf{3}$ and 4 such as an oxygenated methine group $\left(\delta_{\mathrm{H}-3} 4.48, \delta_{\mathrm{C}-3} 80.4\right), 8$ singlet methyl proton signals in high field zone and an acyl group -OCO-R at $\mathrm{C}-3$ via the correlation between $\mathrm{H}-3$ and the resonant peak at $\delta 173.7(\mathrm{C}=\mathrm{O}, \mathrm{C}-1$ ') However, 5 had one more carboxyl carbon at $\delta 180.3$ (-COO-, C-28) and one more oxygenated quaternary carbon at $\delta 91.8$ (>C-O, C-13) instead of two olefinic carbon signals of
C-12 and C-13 as normal. Besides that, the IR spectrum showed a strong absorption at $v 1755$ $\mathrm{cm}^{-1}$ of $\mathrm{C}=\mathrm{O}$ (lactone), so the olefinic carbon at C-13 changed to the oxygenated carbon and created a lactone ring with the carboxyl group (C-28). This was confirmed by the correlation signals of $\mathrm{H}-11, \mathrm{H}-12$ and $\mathrm{H}-18$ with a resonant peak at $\delta 91.8(\mathrm{C}-13)$ and the correlation signals of $\mathrm{H}-16$ with a resonant peak at $\delta 180.3$ (C-28). The long side chain ester at C-3 was determined by MS. The ESI-MS showed a molecular ion peak at $\mathrm{m} / \mathrm{z}=716.5585$ $\left[\mathrm{M}+\mathrm{H}_{2} \mathrm{O}+\mathrm{NH}_{4}\right]^{+}$corresponding to the molecular formula of $\mathrm{C}_{45} \mathrm{H}_{76} \mathrm{O}_{4}+\mathrm{H}_{2} \mathrm{O}+\mathrm{NH}_{4}$ (Calcd for $\mathrm{C}_{45} \mathrm{H}_{76} \mathrm{O}_{4}+\mathrm{H}_{2} \mathrm{O}+\mathrm{NH}_{4}$ 716.6139), so the alcanoyl group shoud be $-\mathrm{CO}-\left(\mathrm{CH}_{2}\right)_{13}-\mathrm{CH}_{3}$. Moreover, the comparison of ${ }^{13} \mathrm{C}-\mathrm{NMR}$ data of the aglycone of 5 with those of literature ${ }^{[3]}$ showed good compatibility. So this compound was determined as 3 $\beta$-pentadecanoyloxyolean28(13)-lactone.

Table 1. NMR data of compounds (1), (2), (3), (4), (5), (6), (8), (9) and (10)

\begin{tabular}{|c|c|c|c|c|c|c|c|c|c|}
\hline $\mathbf{N}^{\mathbf{0}}$ & $\mathbf{1}$ & $\mathbf{2}$ & $\mathbf{3}$ & $\mathbf{4}$ & $\mathbf{5}$ & $\mathbf{6}$ & $\mathbf{8}$ & $\mathbf{9}$ & $\mathbf{1 0}$ \\
\hline 1 & 15.83 & 38.64 & 38.30 & 38.10 & 38.63 & 40.79 & 37.05 & 37.29 & 36.80 \\
2 & 35.25 & 27.25 & 23.60 & 23.59 & 23.65 & 20.16 & 31.12 & 28.92 & 29.23 \\
3 & 72.78 & 79.02 & 80.57 & 80.60 & 80.40 & 38.27 & 76.23 & 71.82 & 70.07 \\
4 & 49.22 & 38.80 & 37.78 & 37.76 & 37.84 & 44.76 & 40.29 & 39.81 & 39.33 \\
5 & 37.88 & 55.21 & 55.28 & 55.34 & 55.09 & 46.64 & 46.71 & 140.79 & 140.10 \\
6 & 41.78 & 18.38 & 18.26 & 18.20 & 17.59 & 18.48 & 26.68 & 121.71 & 121.19 \\
7 & 17.58 & 32.62 & 32.55 & 32.58 & 33.48 & 29.70 & 117.53 & 31.70 & 31.34 \\
8 & 53.24 & 39.82 & 39.84 & 39.32 & 42.20 & 42.28 & 139.09 & 31.99 & 31.34 \\
9 & 37.15 & 47.62 & 47.53 & 47.58 & 50.59 & 155.97 & 49.69 & 50.21 & 49.58 \\
10 & 61.41 & 36.96 & 36.85 & 37.02 & 36.78 & 38.82 & 34.87 & 36.54 & 36.17 \\
\hline
\end{tabular}




\begin{tabular}{|l|c|c|c|c|c|c|c|c|c|}
\hline 11 & 35.60 & 23.60 & 23.55 & 23.42 & 31.49 & 114.92 & 21.40 & 21.10 & 20.56 \\
12 & 30.67 & 122.39 & 122.30 & 122.58 & 18.18 & 37.93 & 39.59 & 39.72 & 38.29 \\
13 & 39.71 & 144.24 & 144.25 & 143.62 & $\mathbf{9 1 . 7 6}$ & 41.27 & 43.42 & 42.25 & 41.82 \\
14 & 38.41 & 41.76 & 41.75 & 40.97 & 42.25 & 44.97 & 54.98 & 56.90 & 56.15 \\
15 & 32.87 & 25.59 & 25.57 & 27.70 & 26.53 & 50.34 & 22.93 & 24.38 & 23.82 \\
16 & 36.12 & 22.06 & 22.04 & 22.91 & 20.85 & 158.54 & 27.96 & 28.26 & 27.74 \\
17 & 30.06 & 36.96 & 36.97 & 46.57 & 44.05 & 105.47 & 56.05 & 56.01 & 55.41 \\
18 & 42.88 & 42.37 & 42.38 & 41.60 & 50.37 & 184.33 & 11.86 & 19.40 & 11.62 \\
19 & 35.37 & 46.51 & 46.46 & 45.88 & 37.45 & 28.25 & 14.14 & 12.01 & 19.04 \\
20 & 28.20 & 31.05 & 30.96 & 30.67 & 31.35 & 23.60 & 36.20 & 40.48 & 35.44 \\
21 & 32.38 & 34.12 & 34.12 & 33.83 & 34.18 & & 18.86 & 21.10 & 18.57 \\
22 & 39.31 & 31.10 & 31.06 & 32.47 & 31.90 & & 34.70 & 138.31 & 33.33 \\
23 & 11.63 & 28.11 & 28.07 & 28.08 & 27.92 & & 31.01 & 129.32 & 25.45 \\
24 & 16.41 & 15.59 & 16.76 & 16.73 & 16.49 & & 156.84 & 51.26 & 45.13 \\
25 & 18.27 & 15.52 & 15.57 & 15.38 & 16.04 & & 33.85 & 31.94 & 28.69 \\
26 & 20.13 & 16.76 & 16.77 & 17.19 & 18.32 & & 21.88 & 19.02 & 18.90 \\
27 & 18.65 & 25.95 & 25.92 & 25.92 & 19.60 & & 22.01 & 21.23 & 19.65 \\
28 & 32.12 & 69.70 & 69.71 & 183.99 & 180.29 & & 105.99 & 25.41 & 22.59 \\
29 & 35.04 & 33.20 & 33.19 & 33.06 & 33.28 & & 15.16 & 12.31 & 11.74 \\
30 & 31.82 & 23.60 & 23.60 & 25.59 & 23.87 & & & & \\
1 & & & 173.70 & 173.72 & 173.69 & & & & 100.79 \\
2 & & & 34.88 & 34.87 & 34.83 & & & & 73.43 \\
3 & & & $14.11^{*}$ & $14.11^{*}$ & $14.09 *$ & & & & 76.74 \\
4 & & & & & & & & & 76.69 \\
5 & & & & & & & & & 76.93 \\
6 & & & & & & & & & 61.06 \\
\hline & $*:$ terminus methyl groups & & & & & & \\
\hline
\end{tabular}

Compound (6) was a diterpen with 20 carbons in the ${ }^{13} \mathrm{C}$-NMR spectrum. It had four olefinic carbon signals of two olefinic bonds at $158.5(=\underline{\mathbf{C}}<, \mathrm{C}-16)$ and $105.5\left(=\underline{\mathbf{C H}}_{2}, \mathrm{C}-17\right)$; $155.9(=\underline{\mathbf{C}}<, \mathrm{C}-9)$ and $114.9(=\underline{\mathbf{C}} \mathrm{H}-, \mathrm{C}-11)$ of a kauran skeleton, one carboxyl carbon signal of a $-\mathrm{COOH}$ group at $\delta 184.3$ and three singlet methyl carbon signals in high field zone. So $\mathbf{6}$ was determined as kaura-9(11),16-dien-18-oic acid (or grandiflorenic acid) by comparison with the published data. ${ }^{[6]}$ 


\section{CONCLUSION}

From flower of Wedelia trilobata Hitch.

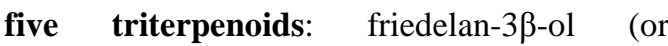
epifriedelanol) (1), erythrodiol (2), 28hydroxy-3 $\beta$-tetradecanoyloxyolean-12-ene (or $3 \beta$-tetradecanoyloxyerythrodiol) (3), $\quad 3 \beta$ henicosanoyloxyolean-12-en-28-oic acid (4), $3 \beta$-pentadecanoyloxyolean-28(13)-lactone (5), one diterpenoid: kaura-9(11),16-dien-18-oic acid (or grandiflorenic acid) (6), one hydroquinone derivative: 2-(3-hydroxy3,7,11,15-tetramethylhexadecyl)-3,5,6trimethyl-1,4-dihydroxybenzene tocopherolhydroquinone) (7) and three sterols: gramisterol (8), stigmasterol (9) and $\beta$ sitosterol 3-O- $\beta$-D-glucopyranoside (10) were isolated. Among them, 2, 5, 7, 8 were found for the first time in Wedelia species.

\title{
MộT SỐ HợP CHẤT TÙ HOA CÂY SƠN CÚC BA THÙY WEDELIA TRILOBATA (L.) HITCH., HỌ CÚC (ASTERACEAE)
}

\author{
Trương Thị Hồng Nguyệt, Nguyễn Thị Hoài Thu, \\ Tôn Thất Quang, Nguyễn Kim Phi Phụng \\ Trường Đại học Khoa học Tự nhiên, ĐHQG HCM
}

TÓM TĂT: Cây Son cúc ba thùy, Wedelia trilobata Hitch., chura được nghiên cứu nhiều trên thế

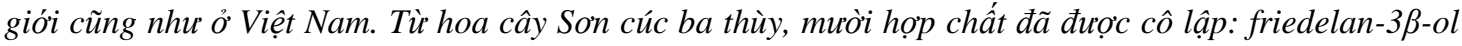

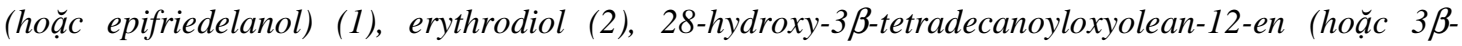

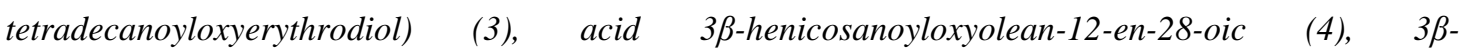
pentadecanoyloxyolean-28(13)-lacton (5), acid kaura-9(11),16-dien-18-oic (hoạc acid grandiflorenic) (6), 2-(3-hydroxy-3,7,11,15-tetrametylhexadecyl)-3,5,6-trimetyl-1,4-dihydroxybenzen (hoạc tocopherolhydroquinon) (7), gramisterol (8), stigmasterol (9) and $\beta$-sitosterol 3-O- $\beta$-D-glucopyranosid (10). Cấu trúc của nhũng hợp chất này được xác định dựa trên dũ liệu phổ NMR và kết hợp với so sánh tài liệu tham khảo.

Tù khóa: Ho Cúc, Wedelia trilobata, triterpen, diterpen, sterol.

\section{REFERENCES}

[1]. Vo Van Chi, "Dictionary of the common plants", Science and Technology
Publishing House, Ha Noi, 2585-2587 (1996).

[2]. Nguyen Thanh Hoang, Nguyen Kim Phi Phung, "Chemical examination of Wedelia biflora (L.) DC, family Asteraceae", 
Proceedings of the Third National

Conference of Science and Technology of

Organic Chemistry, National University-

Ha Noi Publishing House, 348-352 (2005).

[3]. Nguyen Thi Anh Tuyet, Nguyen Tan

Thien, Nguyen Kim Phi Phung,

"Contribution to the study on chemical constituents of Polyscias fruticosa (L.) Harms, (Araliaceae)", Jounal of Chemistry, 43(5), 642-627 (2005).

[4]. Charles J. Pouchert, The Aldrich library of ${ }^{13} \mathrm{C}$ and ${ }^{1} \mathrm{H}$ ft NMR spectra, Aldrich Chemical Company Inc. (1993).

[5]. L. John Goad, Toshihiro Akihisa, “Analysis of sterols”, Blackie Academic \&
Professional, London, New York, Tokyo (1997).

[6]. R. Batista, F. C. Braga, A. B. Oliveira, "Quantitative determination by HPLC of ent-kaurenoic and grandiflorenic acid in aerial parts of Wedelia paludosa D.C", Revista Brasileira de Farmacognosia, Brazilian Journal of Pharmacognosy, 15(2), 119-125 (2005).

[7]. Shashi B. Mahato, Asish P. Kyndu, ${ }^{، 13}$ CNMR spectra of pentacyclic triterpenoids A compilation and some salient features", Phytochemistry, 37(6), 1517-1575 (1994). 